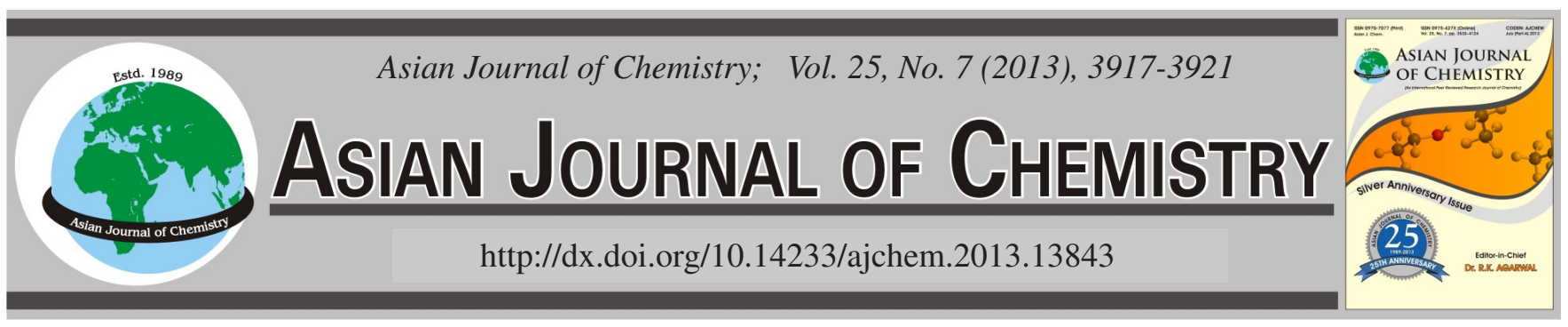

\title{
Chemical Composition of the Essential oil of Brocchia cinerea Grown in South Eastern of Algeria
}

\author{
M. BOUZIANE ${ }^{1,2 *}$, Y. BADJAH-HADJ-AHMED ${ }^{3}$ and M. HADJ-MAHAMMED ${ }^{2}$
}

${ }^{1}$ Faculty of Science and Technology and Sciences of matter, University Kasdi Merbah, Ouargla 30000, Algeria

${ }^{2}$ Biogeochemistry Laboratory of desert environments, Faculty of Science and Technology and Sciences of matter, University Kasdi Merbah. Ouargla. 30000, Algeria

${ }^{3}$ Functional Organic Analysis Laboratory, Faculty of Chemistry, University of Science and Technology Houari Boumediene, BP 32, El Alia, 16111 Algiers, Algeria

*Corresponding author: Fax: +213 29711933; E-mail: bmebarka@yahoo.fr

(Received: 4 April 2012;

Accepted: 18 January 2013)

AJC-12723

\begin{abstract}
The aerial parts of Brocchia cinerea collected from the region of Ouargla, which is located in the southeastern of Algeria, were investigated to obtain the essential oil of this plant by hydrodistillation method. The first sample HE1 was obtained by decantation of the distillate while the second HE2 was extracted from the distillate by $n$-hexane. The samples were analyzed by GC-FID on two capillary columns: a non-polar (HP1) and a polar one (Innowax). The HE1 extract was also analyzed by GC-MS on a DB5 column. The two samples presented nearly the same composition, the main components identified in both were: thujone (47.72\%), camphor (10.54\%), santolinatriene ( 8.00 $\%)$, eucalyptol $(6.37 \%)$ and in second order lyratyl acetate $(4.17 \%)$, terpinen-4-ol (2.77\%).
\end{abstract}

Key Words: Asteraceae, Brocchia cinerea, Essential oil, GC-MS.

ᄂ - - - - - - - - - - - - - - - - - - - - - - - - - - -

\section{INTRODUCTION}

Brocchia cinerea (Fig. 1) belongs to the Asteraceae ${ }^{1}$, it grows abundantly after the rain season in the southeastern of Algerian Sahara (Ouargla city) and surroundings $s^{2,3}$, its local name is "chihia". This plant is traditionally used as a remedy to treat various ailments such as spasm, colic, cough and in the same way in the treatment of other broncho-pulmonar affections.

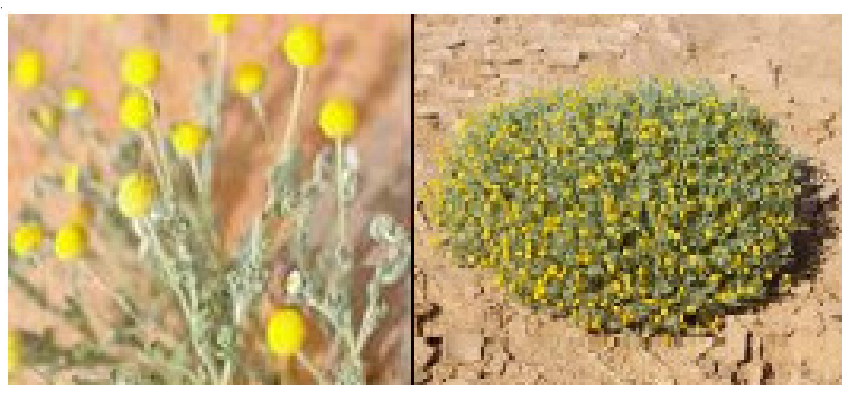

Fig. 1. Brocchia cinerea

In previous studies, it has been demonstrated that the plant showed interesting biological activities: febrifugal, antibacterial, antifungal, analgesic and antiinflammatory ${ }^{4-7}$. Nomad people also add it to tea to perfume it and filter goats butter through it in order to better preserve it.
To the best of our knowledge, no previous investigations were performed about the volatile fraction chemical composition of this plant except a short communication which reported a study of the essential oil of Brocchia cinerea growing in Egypt ${ }^{8}$.

The objectives of the present paper are the determination of the essential oil chemical composition of this plant. Further more a comparison of the chemical composition of the two samples, HE1 and HE2 which are respectively the pure essential oil and the one obtained with $n$-hexane.

\section{EXPERIMENTAL}

Extraction of the essential oils: The plant material of Brocchia cinerea was collected in March 2005, during the flowering stage, at $20 \mathrm{~km}$ north from Ouargla city. Plant identification was carried out by Dr K. Maiza, medical botanist in medical botanic laboratory, faculty of medicine, Algiers University, Algeria.

The aerial parts of Brocchia cinerea were air-dried at room temperature in a dark place. The essential oils were obtained by hydrodistillation using a modified Clevenger-type apparatus for $2 \mathrm{~h}$. The sample HE1 was collected directly by decantation of the distillate and stored while HE2 was obtained by liquid-liquid extraction of the distillate with $n$-hexane. After evaporation of $n$-hexane, both samples HE1 and HE2 were stored at $4{ }^{\circ} \mathrm{C}$.

Analysis of HE1 and HE2: In a first time, HE1, HE2 were analyzed using gas chromarography with flame ionization 
detection (GC-FID) on two types of capillary column: HP1 and innowax. Then, gas chromarography coupled at mass spectrometry (GC-MS) on DB5 capillary column, was used to analyze the samples.

GC-FID analysis: The GC-FID analyses were carried out using an Agilent HP5975 Series C gas chromatograph equipped first with HP1 a fused silica capillary column coated with a non-polar phase $(50 \mathrm{~m} * 200 \mu \mathrm{m}$ id, film thickness 0.33 $\mu \mathrm{m})$. An Innowax column coated with a polar stationary phase $(50 \mathrm{~m} \times 200 \mu \mathrm{m}$ id, film thickness $0.5 \mu \mathrm{m})$ was then used to separate both samples.

With both columns, helium was the carrier gas with a flow rate of $1.3 \mathrm{~cm}^{3} \mathrm{~min}^{-1}$ while the oven temperature was programmed as follows: $80{ }^{\circ} \mathrm{C}$ during $8 \mathrm{~min}$, then increased up to $200{ }^{\circ} \mathrm{C}$ at a $2{ }^{\circ} \mathrm{C} / \mathrm{min}$ rate and finally to $285^{\circ} \mathrm{C}$ at $10^{\circ} \mathrm{C} / \mathrm{min}$. The injector and detector temperatures were $250{ }^{\circ} \mathrm{C}$ and $300{ }^{\circ} \mathrm{C}$, respectively.

GC-MS analysis: An Agilent HP6890 GC-MS system was used equipped with a mass-selective detector using electron impact ionization. The separations were performed on a DB5 capillary column $(30 \mathrm{~m} \times 0.25 \mathrm{~mm}$ id, $0.25 \mu \mathrm{m}$ film thickness), which was directly interfaced into the ion source of the MS system. The oven temperature program used for analysis was as follows: the initial temperature was maintained at $45^{\circ} \mathrm{C}$ for $8 \mathrm{~min}$ and then increased up to $250^{\circ} \mathrm{C}$ at $2{ }^{\circ} \mathrm{C} / \mathrm{min}$ rate. $250^{\circ} \mathrm{C}$ was maintained for $14 \mathrm{~min}$. Helium was the carrier gas at a $0.5 \mathrm{~cm}^{3} \mathrm{~min}^{-1}$ flow rate and the split ratio was (1:80). Both injector and detector temperatures were $250{ }^{\circ} \mathrm{C}$.

The electron impact ionization energy was $70 \mathrm{eV}$ and the mass range scanned was $29-550(\mathrm{~m} / \mathrm{z})$. The retention indices (Ir) for all the compounds were determined according to Van Den Dool method using retention times of $n$-alkanes (C5-C31), that had been injected after the essential oils under the same chromatographic conditions.

The components were first identified by comparison of their retention indices with those reported in published works ${ }^{9-27}$ then confirmed by comparison of their mass spectra with those of standards in Wiley, NIST and aromas CNRS libraries.

\section{RESULTS AND DISCUSSION}

The hydrodistillation of Brocchia cinerea aerial parts, gave a yellow like brown essential oil (HE1) and a pale yellow essential oil (HE2), with a yield of : HE1 (0.75 \% v/w), HE2 $(0.85 \% \mathrm{v} / \mathrm{w})$ this last higher yield could be explained by the presence of $n$-hexane trace.

Data obtained from qualitative and semi-quantitative determinations of the two investigated oil samples are shown in Tables 1 and 2. GC-FID chromatograms are very similar for both samples, as it can be seen in Table-1, except for $\alpha$ terpinene and trans- $\beta$-farnesene which are present in HE1 but

TABLE-1

CHEMICAL COMPOSITION OF HE1 AND HE2 ANALYZED BY GC-FID

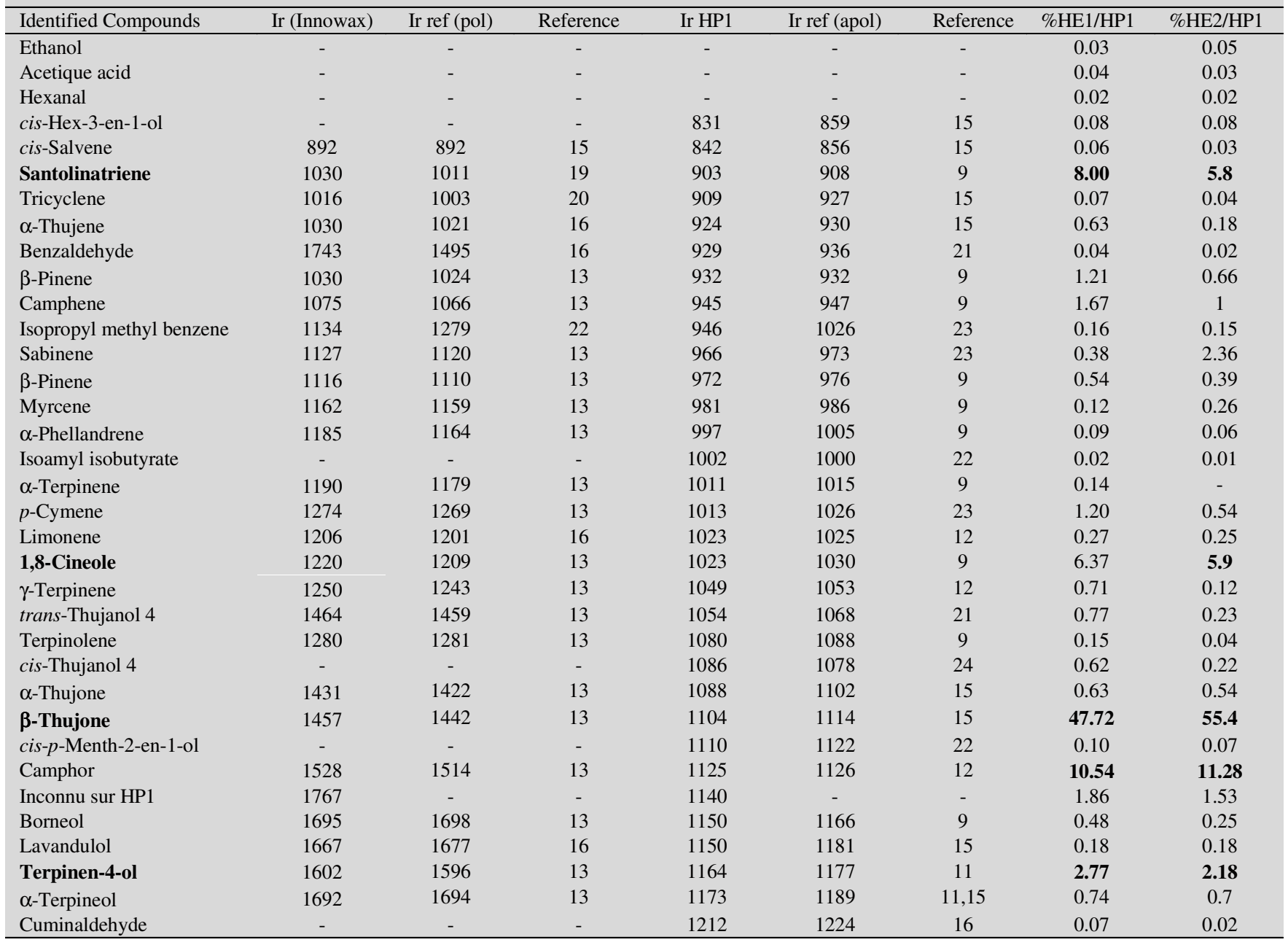




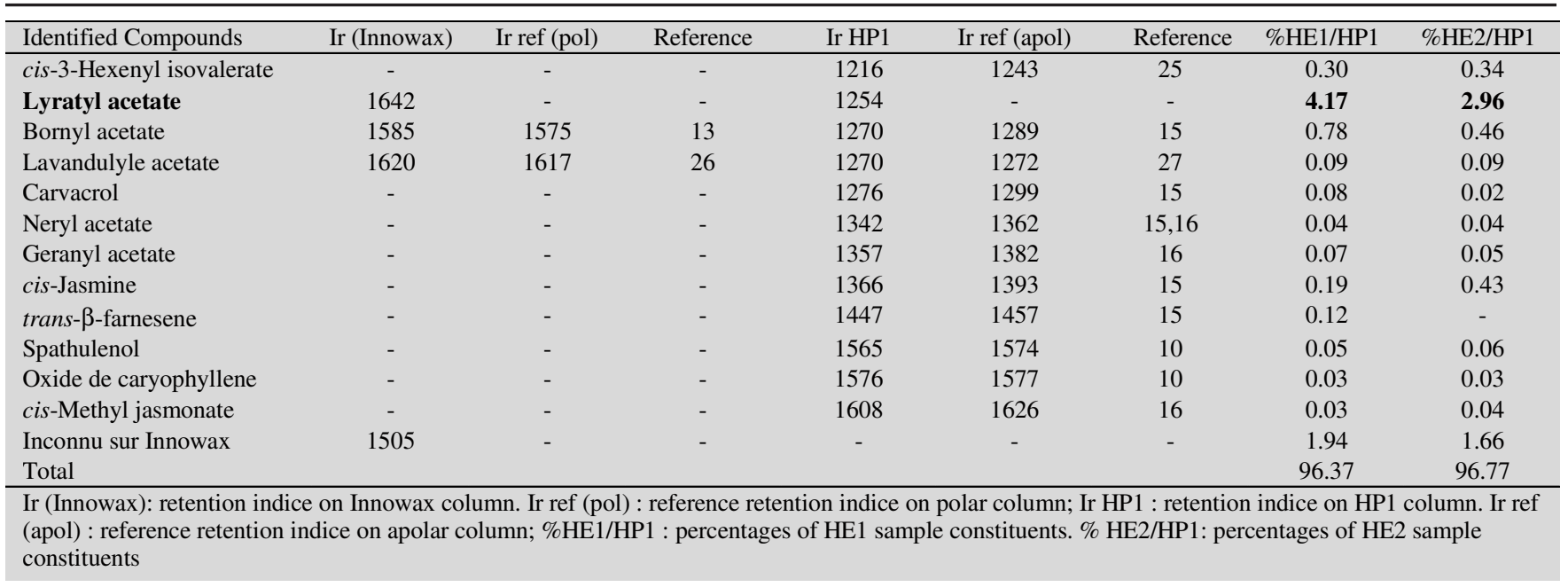

not in HE2. The main compounds were $\beta$-thujone (47.72\%), camphor $(10.54 \%)$, santolinatriene $(8.00 \%)$ eucalyptol $(6.37 \%)$, lyratyl acetate $(4.17 \%)$ and terpinen-4-ol $(2.77 \%)$.

It can be noticed that the relative percentages of most constituents are higher in HE1 than in HE2, while the two main components are more concentrated in HE2. Their relative percentages in HE2 and HE1 are, respectively: 55.4\%-47.72 \% for $\beta$-thujone and $11.28 \%-10.54 \%$ for camphor.

More than $50 \%$ of the chemical composition; including the main compound are also identified by Fournier et al. ${ }^{8}$ but with difference in percentages. That probably returns to the different collection origins of this plant.

As it can be seen on the histogram (Fig. 2), more than $90 \%$ of Brocchia cinerea essential oil is constituted approximately by $30 \%$ of monoterpenic hydrocarbons, $42 \%$ of oxygenated monoterpenes, $4 \%$ of oxygenated sesquiterpenes and $24 \%$ of other substances.

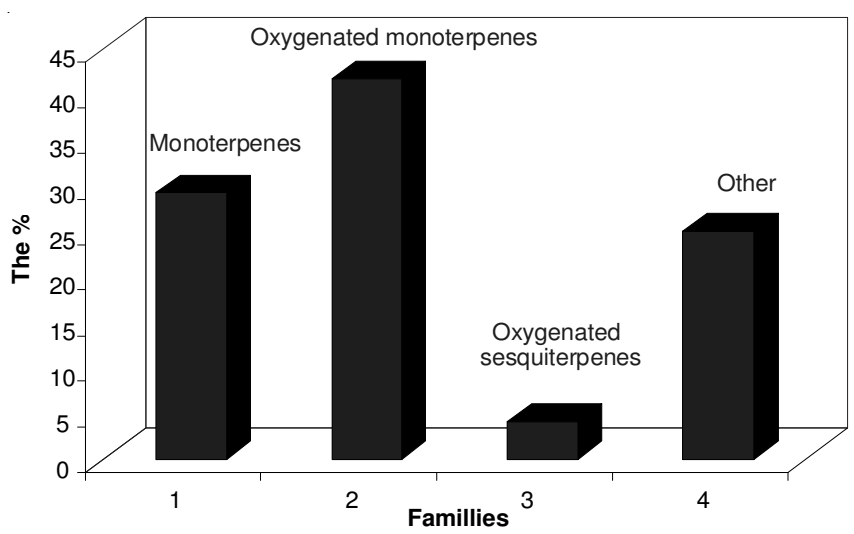

Fig. 2. Histogram of the present chemical families

Regarding the chemical composition of this plant, its interesting biological activities can be explained by the high content of oxygenated monoterpenoids, in particular eucalyptol and camphor, which are well-known for their antiseptic properties and their efficiency in the treatment of respiratory diseases ${ }^{17}$. On the other hand, the relatively high concentration of thujone should be also highlighted, this compound being known as a toxic substance ${ }^{18}$.
Two constituents were detected by GC-FID but could not be identified they were eluted on the Innowax column at the retention indices 1505 and 1767 , while the second one had a retention index of 1140 on the HP1 capillary column.

Because of its high sensitivity, GC-MS was very useful in the qualitative analysis of our samples. More than 50 peaks were detected, among them 32 compounds were identified and reported on Table-2, 21 of them being already detected by GC-FID analysis.

The results show that the main compounds are $\alpha$-thujone, camphor and eucalyptol. Other characteristic terpenic substances were identified in this work such as $p$-menth-1-en-8ol, sabinene, $\alpha$-thujenal, trans-piperitol and $\beta$-pinene.

$\beta$-Thujone was not detected by GC-MS, but $\alpha$-thujone was present with a relatively high percentage, as it can be seen on the chromatogram shown in Fig. 3.

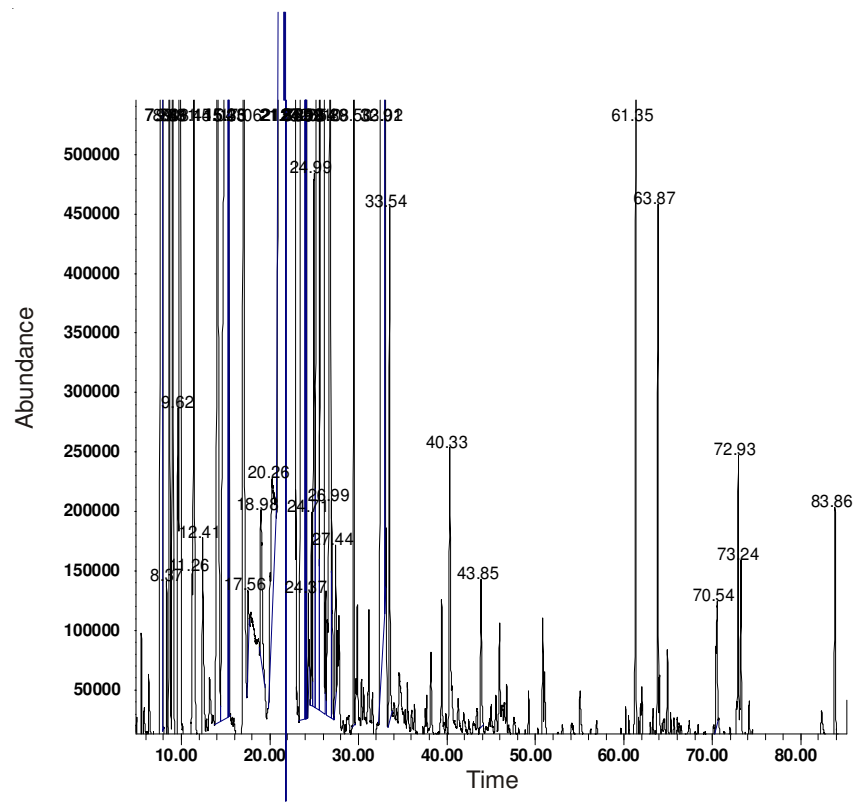

Fig. 3. Chromatogram obtained by GC-MS

It is important to notice that 21 other constituents were also detected by this analysis. Among these non-identified components, some of them are present in relatively high concentration as it can be seen on the chromatogram (Fig. 3). 
TABLE-2

CHEMICAL COMPOSITION OF HE1 ANALYZED BY GC-MS

\begin{tabular}{|c|c|c|c|c|}
\hline Identified compounds & $\operatorname{tr}(\min )$ & $\operatorname{Ir}(\exp )$ & MS (exp) & MS (refe) \\
\hline Hexanal & 4.24 & 800 & $\begin{array}{l}\text { 44(100), 56(79), 41(60), 43(55), 29(35), } \\
27(33), 72(15)\end{array}$ & $\begin{array}{l}\text { 44(100), 56(82), 41(66), 43(53), 29(38), } \\
27(33), 72(19)\end{array}$ \\
\hline cis-Salvene & 5.5 & 837 & $\begin{array}{l}41(100), 67(98), 81(96), 68(80), 69(60), \\
55(50), 39(45), 109(40)\end{array}$ & $\begin{array}{l}41(100), 67(72), 81(64), 55(38), 109(22), \\
95(10), 124(1)\end{array}$ \\
\hline Isoamyl acetate & 6.33 & 864 & $\begin{array}{l}43(100), 70(45), 55(42), 41(20), 42(18), \\
32(12), 61(10)\end{array}$ & $\begin{array}{l}43(100), 55(43), 73(38), 41(37), 42(33), \\
61(27)\end{array}$ \\
\hline Santolinatrienne & 7.88 & 907 & $\begin{array}{l}93(100), 121(80), 79(70), 91(50), 77(47), \\
67(40), 105(32), 41(25)\end{array}$ & $\begin{array}{l}93(100), 41(72), 79(70), 121(55), 77(51), \\
67(49), 53(34)\end{array}$ \\
\hline Tricyclene & 8.36 & 916 & $\begin{array}{l}\text { 93(100), 79(32), 91(30), 79(32), 92(30), } \\
77(30), 41(29), 121(25), 136(20), 39(20)\end{array}$ & $\begin{array}{l}93(100), 91(31), 92(27), 79(25), 41(24), \\
39(22), 77(22), 121(20) .\end{array}$ \\
\hline$\alpha$-Thujene & 8.68 & 921 & $\begin{array}{l}93(100), 91(60), 77(47), 92(43), 79(15), \\
136(15), 41(10)\end{array}$ & $\begin{array}{l}93(100), 77(23), 91(21), 92(20), 41(15) \\
39(14), 79(10), 121(5)\end{array}$ \\
\hline$\alpha$-Pinene & 9.08 & 928 & $\begin{array}{l}\text { 93(100), 91(35), 92(32), 77(29), 79(25), } \\
121(19), 41(15), 39(10)\end{array}$ & $\begin{array}{l}93(100), 92(35), 91(32), 77(29), 79(23), \\
41(20), 39(19), 121(14)\end{array}$ \\
\hline Camphene & 9.91 & 942 & $\begin{array}{l}93(100), 121(75), 79(40), 91(37), 67(35), \\
107(32), 77(30), 41(20) 39(18), 136(15)\end{array}$ & $\begin{array}{l}93(100), 121(58), 79(40), 91(37), 39(34), \\
41(32), 67(29), 77(28)\end{array}$ \\
\hline$\beta$-Pinene & 11.45 & 968 & $\begin{array}{l}93(100), 69(40), 41(39), 79(37), 77(36), \\
91(36), 121(18), 136(15)\end{array}$ & $\begin{array}{l}93(100), 41(61), 69(35), 39(32), 91(31), \\
77(28), 79(27), 27(21)\end{array}$ \\
\hline$\alpha$-Phellandrene & 13.19 & 998 & $\begin{array}{l}\text { 93(100), 91(60), 77(55), 92(40), 136(30), } \\
41(25), 32(22), 39(20), 57(18), 79(18), 85(15)\end{array}$ & $\begin{array}{l}93(100), 91(33), 77(31), 91(25), 136(16), \\
41(14), 39(11), 27(10)\end{array}$ \\
\hline Eucalyptol & 15.30 & 1027 & $\begin{array}{l}43(100), 81(98), 108(90), 154(85), 111(80) \text {, } \\
139(75), 71(70), 84(68), 93(65), 55(50)\end{array}$ & $\begin{array}{l}43(100), 93(55), 81(55), 71(47), 69(40), \\
84(38), 68(38), 108(36)\end{array}$ \\
\hline$\gamma$-Terpinene & 17.06 & 1053 & $\begin{array}{l}93(100), 91(50), 77(35), 136(35), 121(30), \\
43(20), 39(10)\end{array}$ & $\begin{array}{l}93(100), 91(33), 77(32), 136(29) \\
121(27), 39(23), 43(23), 27(23)\end{array}$ \\
\hline$\alpha$-Thujone & 21.48 & 1115 & $\begin{array}{l}110(100), 81(95), 95(80), 67(60), 69(57) \\
68(55), 109(55), 41(48)\end{array}$ & $\begin{array}{l}110(100), 81(88), 95(70), 67(68), 68(58), \\
41(58), 69(53), 109(43)\end{array}$ \\
\hline $\begin{array}{l}\text { 8-Methylene-3-oxatri- } \\
\text { cyclo[5,2,0,0(2,4)]nonane }\end{array}$ & 22.88 & 1136 & $\begin{array}{l}79(100), 91(95), 96(75), 77(73), 39(55), \\
109(55), 81(50), 41(50), 69(53), 93(45)\end{array}$ & $\begin{array}{l}79(100), 92(83), 39(69), 91(62), 77(57), \\
41(50), 27(47), 93(41)\end{array}$ \\
\hline Camphor & 23.92 & 1150 & $\begin{array}{l}95(100), 81(82), 108(45), 69(40), 152(42), \\
55(35), 41(38)\end{array}$ & $\begin{array}{l}95(100), 41(73), 81(74), 108(39), 69(39), \\
55(38), 27(35), 39(34)\end{array}$ \\
\hline$\alpha$-Thujenal & 26.29 & 1186 & $\begin{array}{l}79(100), 77(40), 107(35), 105(30), 108(18), \\
106(17), 91(17), 41(15), 43(14)\end{array}$ & $\begin{array}{l}79(100), 107(51), 77(33), 105(29) \\
108(29), 106(28), 41(17), 43(16)\end{array}$ \\
\hline p-Cymen-8-ol & 26.53 & 1189 & $\begin{array}{l}43(100), 135(85), 91(27), 67(20), 39(15), \\
41(17), 79(18), 55(12), 82(9), 105(7)\end{array}$ & 43(100), 135(52), 91(21), 39(9), \\
\hline$p$-menth-1-en-8-ol & 26.89 & 1194 & $\begin{array}{l}\text { 59(100), 93(60), 121(49), 136(45), 81(33), } \\
43(31), 67(20)\end{array}$ & $\begin{array}{l}59(10), 93(60), 121(49), 136(45), 81(33), \\
43(31), 67(16), 92(17)\end{array}$ \\
\hline trans-Piperitol & 27.82 & 1207 & $\begin{array}{l}84(100), 83(40), 41(35), 139(32), 55(25), \\
91(20), 79(20), 67(15)\end{array}$ & $\begin{array}{l}84(100), 41(27), 83(27), 136(26), 93(25), \\
43(22), 55(20), 77(18), 69(17), 67(8)\end{array}$ \\
\hline cis-3-Hexenylisovalerate & 29.5 & 1232 & $\begin{array}{l}82(100), 67(99), 57(98), 41(35), 85(32), \\
55(20), 54(9)\end{array}$ & $\begin{array}{l}82(100), 67(77), 57(73), 85(55), 41(48), \\
55(27), 43(22), 29(22)\end{array}$ \\
\hline Lyratyl acetate & 32.91 & 1280 & $\begin{array}{l}\text { 119(100), 43(67), 91(50), 93(47), 79(45), } \\
77(38), 105(35), 134(22)\end{array}$ & $\begin{array}{l}119(100), 43(67), 134(47), 93(42) \\
91(41), 79(34), 105(26), 77(22)\end{array}$ \\
\hline Bornyl acetate & 33.02 & 1281 & $\begin{array}{l}95(100), 43(58), 93(48), 121(45), 136(40), \\
41(20), 80(18), 55(18)\end{array}$ & $\begin{array}{l}95(100), 43(76), 93(45), 136(39) \\
121(34), 41(27), 80(17), 55(15)\end{array}$ \\
\hline Lavandulyl acetate & 33.53 & 1289 & $\begin{array}{l}\text { 69(100), 43(85), 93(75), 41(65), 68(45), } \\
91(25), 121(23), 67(20), 136(10)\end{array}$ & $\begin{array}{l}69(100), 43(74), 93(73), 41(50), 68(46), \\
121(24), 67(20), 136(12)\end{array}$ \\
\hline Carvacrol & 34.04 & 1296 & $\begin{array}{l}\text { 135(100), 150(35), 91(38), 43(48), 32(45), } \\
79(28), 77(25), 107(19), 115(18), 136(10)\end{array}$ & $\begin{array}{l}\text { 135(100), 150(31), 91(13), 136(10), } \\
77(7), 107(7), 117(6), 115(5)\end{array}$ \\
\hline Neryl acetate & 38.19 & 1359 & $\begin{array}{l}\text { 69(100), 43(67), 41(65), 3(45), 68(40), } \\
67(27), 121(25), 80(19)\end{array}$ & $\begin{array}{l}69(100), 41(67), 43(45), 68(37), 93(35), \\
67(18), 80(13), 121(11)\end{array}$ \\
\hline Geranyl acetate & 39.43 & 1379 & $\begin{array}{l}\text { 69(100), 43(65), 41(62), 68(45), 93(24), } \\
121(18), 138(14), 67(20)\end{array}$ & $\begin{array}{l}\text { 69(100), 43(65), 41(63), 68(49), 93(24), } \\
136(22), 121(14), 67(13)\end{array}$ \\
\hline cis-Jasmone & 40.33 & 1392 & $\begin{array}{l}79(100), 91(75), 110(75), 164(68), 55(65), \\
\text { 149(62), 41(61), 93(60), 77(60), 122(58), } \\
135(58)\end{array}$ & $\begin{array}{l}79(100), 91(98), 39(97), 77(64), 164(60), \\
91(58), 93(54), 53(50), 55(49), 110(45), \\
149(42), 135(40), 122(40)\end{array}$ \\
\hline Spathulenol & 50.82 & 1566 & $\begin{array}{l}\text { 43(100), 41(63), 91(62), 119(57), 205(50), } \\
93(48), 105(48), 159(38)\end{array}$ & $\begin{array}{l}43(100), 41(63), 205(61), 119(57) \\
91(50), 93(48), 159(43), 105(40)\end{array}$ \\
\hline Caryophyllène oxide & 51.04 & 1570 & $\begin{array}{l}43(100), 41(97), 79(82), 93(70), 91(54), \\
69(45), 55(42), 109(35), 121(25), 135(10)\end{array}$ & $\begin{array}{l}43(100), 41(97), 79(82), 93(70), 91(54), \\
69(45), 55(42), 109(35), 121(25), 135(10)\end{array}$ \\
\hline Methyl jasmonate & 55.05 & 1643 & $\begin{array}{l}83(100), 41(58), 151(40), 67(40), 95(40), \\
55(43), 93(35), 109(38)\end{array}$ & $\begin{array}{l}83(100), 41(53), 151(46), 67(33), 95(32) \\
55(29), 93(28), 109(28)\end{array}$ \\
\hline
\end{tabular}




\section{Conclusion}

This study about the chemical composition of the plant Brocchia cinerea species collected from the region of Ouargla, southeastern Algeria, was carried by extraction of its essential oil which has a rich and varied chemical composition; $30 \%$ of monoterpenic hydrocarbons, $42 \%$ of oxygenated monoterpenes, $4 \%$ of oxygenated sesquiterpenes and $24 \%$ of other substances. $n$-Hexane has proved to be a good solvent for this purpose. It allowed the identification of most constituents of Brocchia cinerea essential oil.

The high level of oxygenated monoterpenoids, such as eucalyptol and camphor, confirms the antimicrobial and bactericidal properties of this plant and the importance of further investigations to evaluate its potential applications.

\section{REFERENCES}

1. P. Ozenda, Flore et végétation du Sahara, CNRS édition, Paris (1993)

2. K. Maiza, R.A. Brac de La Perrière, V. Hammiche, 2th European Ethnopharmacology Colloquium and $11^{\text {th }}$ International Ethnomedicine Conference, Heidelberg (Germany) pp. 24-27 (March 1993).

3. V. Hammiche and K. Maiza, Ethnopharmacolology, 105, 358 (2006).

4. M. Larhsini, M. Markouk, J.T. Jaouhari, K. Bekkouche, H.B. Lazrek and M. Jana, Phytotherapy Res., 16(S1), 97 (2002).

5. M. Markouk, A. Radwane, H.B. Lazrek, M. Jana and A. Benjama, Fitotherapia, 70, 314 (1999)

6. M. Markouk, H.B. Lazrek, M. Larhsini, K. Bekkouche and M. Jana, Therapie, 54, 759 (1999).

7. M. Markouk, H.B. Lazrek and M. Jana, Phytother Res., 13, 229 (1999).

8. G. Fournier, H. Baghdadi, S.S. Ahmed and M. Paris, Planta Medica, 55, 580 (1989)

9. K. Javidnia, R. Miri, M. Kamalinejad and A. Jamalian, Flav. Fragr. J., 19, 213 (2004).
10. I. Schwob, J.M. Bessière and J. Viano, Comptes Rendus Biol., 325, 781 (2003).

11. F. Sahin, M. Gulluce, D. Daferera, A. Sökmen, M. Sökmen, M. Polissiou, G. Agar and H. Ozer, Food Control, 15, 549 (2004).

12. C. Shang, Y. Hu, C. Deng and K. Hu, J. Chromatogr. A., 942, 283 (2002).

13. Revue des régions arides (numéro spécial), Actes du Séminaire International sur les Plantes à Parfum Aromatiques et Médicinales (SIPAM 2006), 2-4 November 2006, Jerba (Tunisia).

14. S.J. Lee, K. Umamo, T. Shibamoto and K.G. Lee, Food Chem., 91, 131 (2005).

15. R.P. Adams, Identification of Essential Oil Components by Gas Chromatography/Quadrupole Mass Spectroscopy. Academic Press, New York, Appendix I, II (1989).

16. http://www.flavornet.org/d_kovats_ov101.html Accessed 10/06/ 2008.

17. A. Sökmen, G. Vandar-Ünlu, M. Polissiou, D. Daferera, M. Sökmen and E. Dönmez, Phytother Res., 7, 1005 (2003).

18. K.M. Höld, N.S. Sirisoma, T. Ikeda, T. Narahashi and J.E. Casida, Proc. Natl. Acad. Sci. U.S.A., 97, 3826 (2000).

19. A. Muselli, P.G. Rossi, M.J. Desjobert, A.F. Bernardini, L. Berti and J. Costa, Flav. Fragr. J., 22, 217 (2007).

20. G.R. Mallavarapu, B.R. Rrao, P.N. Kaul, S. Ramesh and A.K. Bhattacharya, Flav. Fragr. J., 13, 167 (1998).

21. J.P. Mevy, J.M. Bessiere, S. Greff, G. Zombre and J. Viano, J. Biochem. Syst. Ecol., 34, 549 (2006).

22. S.K. Srivastava, A. Ahmad, K.V. Syamsunder, K.K. Aggarwal and S.P.S. Shanuja, Flav. Fragr. J., 18, 361 (2003).

23. R.P. Adams, M.S. Gonzalez Elizondo, M. Gonzalez Elizondo and E. Slinkman, J. Biochem. Syst. Ecol., 34, 205 (2006).

24. A.H. El-Ghorab, A.F. Mansour and K.F. El-Masry, Flav. Fragr. J., 19, 54 (2004).

25. S. Maccioni, R. Baldini, P.L. Cioni and G. Flamini, Flav. Frag. J., 22, 61 (2007).

26. N. Tabanca, B. Demirci, K.H.C. Baser, E. Mincsovics, S.I. Khan, M.R. Jacob and D.E. Wedge, J. Chromatogr. B., 850, 221 (2007).

27. J.J. Filippi, D.A. Lanfranchi, S. Prado, N. Baldovini and U.J. Meierhenrich, J. Agric. Food Chem., 54, 6308 (2006). 